\author{
C. Faldini \\ M. Manca \\ S. Pagkrati \\ D. Leonetti \\ M. Nanni \\ G. Grandi \\ M. Romagnoli \\ M. Himmelmann
}

Received: 3 November 2005

Accepted: 3 December 2005

C. Faldini $(\bowtie) \cdot$ S. Pagkrati • D. Leonetti

M. Nanni • G. Grandi • M. Romagnoli

Department of Orthopaedic Surgery

University of Bologna

Istituto Ortopedico Rizzoli

Via G. Pupilli 1, I-40136 Bologna, Italy

E-mail: cesare.faldini@ior.it

\section{Manca}

Department of Orthopaedic Surgery

Massa Carrara Hospital, Italy

M. Himmelmann

Scuola Superiore S. Anna

University of Pisa, Italy

\title{
Surgical treatment of complex tibial plateau fractures by closed reduction and external fixation. A review of 32 consecutive cases operated
}

\begin{abstract}
Complex tibial plateau fractures are a challenge in trauma surgery. In these fractures it is necessary to anatomically reduce the articular part of the fracture and to obtain stable fixation. The aim of this study is to review the results of a surgical technique consisting of fluoroscopic closed reduction and combined percutaneous internal and external fixation. Thirty-two complex tibial plateau fractures in 32 patients were included. Twenty-one fractures were closed, 4 were open Gustilo grade I, 3 were Gustilo grade II and 4 were Gustilo grade III. The mean age was 37.8 years (range 21-64 years). Surgery was performed with patients in transcalcaneal traction and the knee flexed at $30^{\circ}$ was used. Through a $1-\mathrm{cm}$ incision centred over the tibial metaphysis of the tibia, a $3.2-\mathrm{mm}$ hole was drilled in the antero-medial tibial aspect. The tibial plateau fracture fragments were elevated using either 1 or 2 curved Kirschner wires under fluoroscopy to control the reduction. Then the

clinically and radiographically evaluated at a mean follow-up of 48 months (range 38-57 months). Clinical results were evaluated according to the Knee Society clinical score. Average healing time was 24 weeks (range 18-29 weeks). In 1 patient a non-union occurred. This patient was treated with open reduction and plate fixation. In 2 patients a varus knee deformity occurred and a surgical correction was performed. There were no surgical complications. Mean knee range of motion was $105^{\circ}$ (range $75-125^{\circ}$ ) and mean Knee Society clinical score was 89 . Twenty-five results were scored as excellent, 4 good, 2 fair and 1 poor. Using this technique there is limited soft tissue damage and virtually no periosteum damage to the fracture fragments. However anatomical reconstruction of the joint can be obtained. Furthermore knee rehabilitation can be started immediately after surgery. We think that these factors were responsible for the optimal clinical long-term results.
\end{abstract} fragments were fixed with 2 cannulated $\mathrm{AO}$ screws inserted through small incisions into the medial aspect of the tibial plateau. Knee rehabilitation started postoperatively. Weight bearing started after 8-12 weeks depending upon the radiographic appearance. All external fixators were removed in outpatient facilities. All patients were
Key words Closed reduction • External fixation - Open fracture • Surgical technique - Tibial plateau fracture 


\section{Introduction}

Complex tibial plateau fractures consist in interruption of one or two tibial condyls, associated with fracture of the proximal metaphysis of the tibia. These complex fractures were classified as C3 by Muller in 1970 [1] or type VI by Schatzkler in 1979 [2]. They are usually caused by highenergy trauma, which often induces soft tissue damage and the surgical strategy may be complicated by the presence of other injuries. For these reasons tibial plateau fractures remains a challenge in trauma surgery and a debated topic.

Conservative treatment by traction, bracing and casting of complex tibial plateau fractures rarely permits an accurate reduction of the articular surface of the tibial plateau. In fact many authors report unsatisfactory results with loss of reduction, mechanical deformities and secondary arthritis [3-8]. In complex tibial plateau fractures, it is necessary to anatomically reduce the articular part of the fracture in order to avoid secondary arthritis, and to obtain a stable articular and metaphyseal fixation promoting fracture healing [1]. These two goals should be obtained with as least an invasive technique as possible [9-11]. Open reduction and internal fixation with plates and screws as described by several authors permits an accurate reduction of the articular surface involved in the trauma, but with a high rate of deep infection, wound dehiscence and soft tissue problems [12, 13]. The use of closed reduction and percutaneous fixation techniques have reduced the incidence of wound complications $[6,8$, $11,14-17]$. This technique is usually sufficient to stabilise the articular fragments after fluoroscopic or arthroscopic reduction, but it is not adequate to control the metaphyseal fragments. For these reasons some authors have suggested surgical techniques based on closed internal fixation of the articular fracture, and circular external fixation of the metaphyseal fracture [17-21].

The aim of this study is to review the results of fluoroscopic closed reduction combined with percutaneous internal and hybrid external fixation of complex tibial plateau fractures.

\section{Materials and methods}

Thirty-two complex tibial plateau fractures in 32 patients were included. All the fractures resulted from high-energy trauma. Twenty-one fractures were closed, 4 were open Gustilo grade I, 3 were Gustilo grade II and 4 were Gustilo grade III [22]. The mean age was 37.8 years (range $21-64$ years). Nineteen patients sustained fractures of other bones and 9 had abdominal or neu- rological involvement. All patients were studied with standard 2 projection radiographs of the entire leg, while the knee was examined with 2 oblique projections. Two fractures were also studied with CT scan. The injured legs were initially treated with transcalcaneal traction. Surgery was performed immediately after admission to the hospital in 18 cases, while in the other 14 cases it was delayed for 7-12 days after trauma, depending on the general condition of the patient.

Surgery was performed under general or spinal anaesthesia. The patient was positioned on the operating table with the knee flexed at $30^{\circ}$ and the leg in transcalcaneal traction. A tourniquet is not a significant advantage in closed reduction, but if used, should be deflated as soon as possible. The fracture was checked with an image intensifier.

Through a small skin incision over the antero-medial aspect of the tibial metaphysis, a small hole was made in the tibial cortex. A blunt tipped curved 3-mm Kirschner wire was inserted through the hole up to the articular fragments (Fig. 1), which were elevated under image intensifier control (Fig. 2). In some cases, more than one Kirschner wire was required to reduce the articular fracture.

Through a small lateral incision, a Kirschner wire was inserted across the tibial plateau to stabilise the reduced fragments and a cannulated screw was introduced over it. Two cannulated screws were usually inserted from the lateral condyle, but position and number of the screws was determined by the fracture type. After reduction of the articular fracture, an Orthofix hybrid external fixator was applied (Fig. 3).

A ring of appropriate size was positioned at the level of the fibular head. All wires were applied in the transversal plane, 2 from lateral to medial, and the remaining 2 from antero-lateral to postero-medial. Each wire was tensioned at $1400 \mathrm{~N}$ and locked to the frame. The metaphyseal fracture was reduced accurately

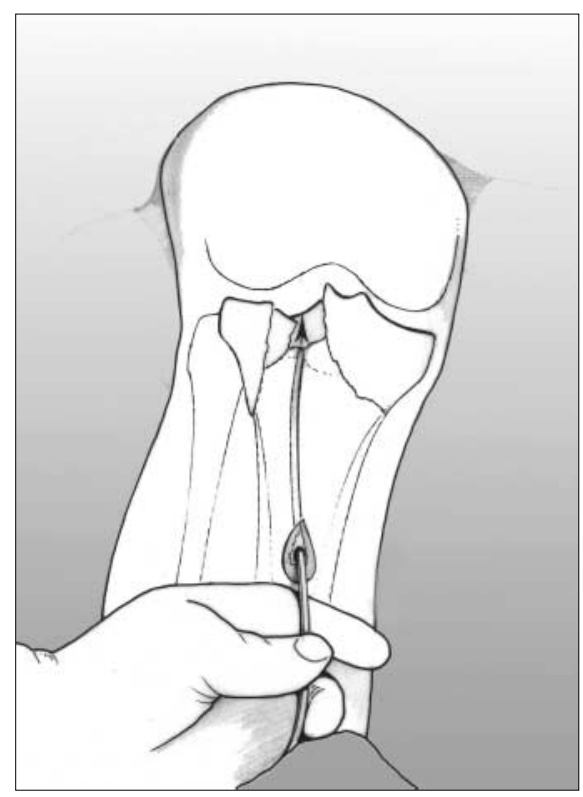

Fig. 1 Through a small hole drilled on the tibial cortex, a blunt tipped Kirschner wire was inserted to reduce the articular fragments of the fracture 

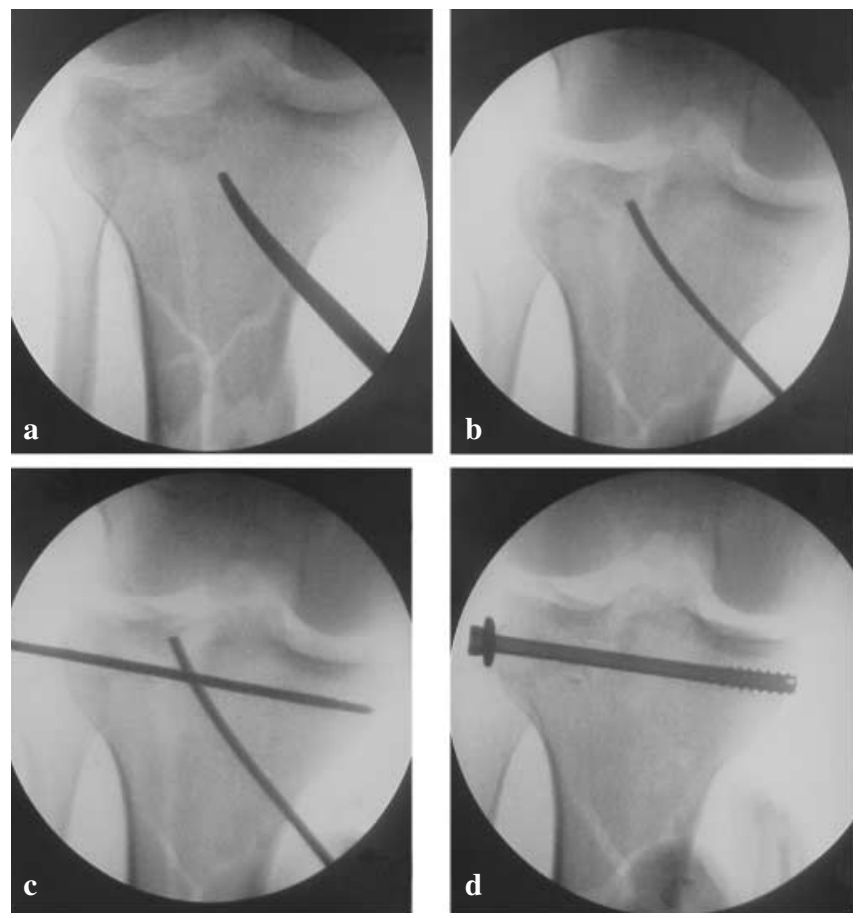

Fig. 2a, b The articular fragment of the fracture was reducted, and $(\mathbf{c}, \mathbf{d})$ stabilised by a cannulated screw

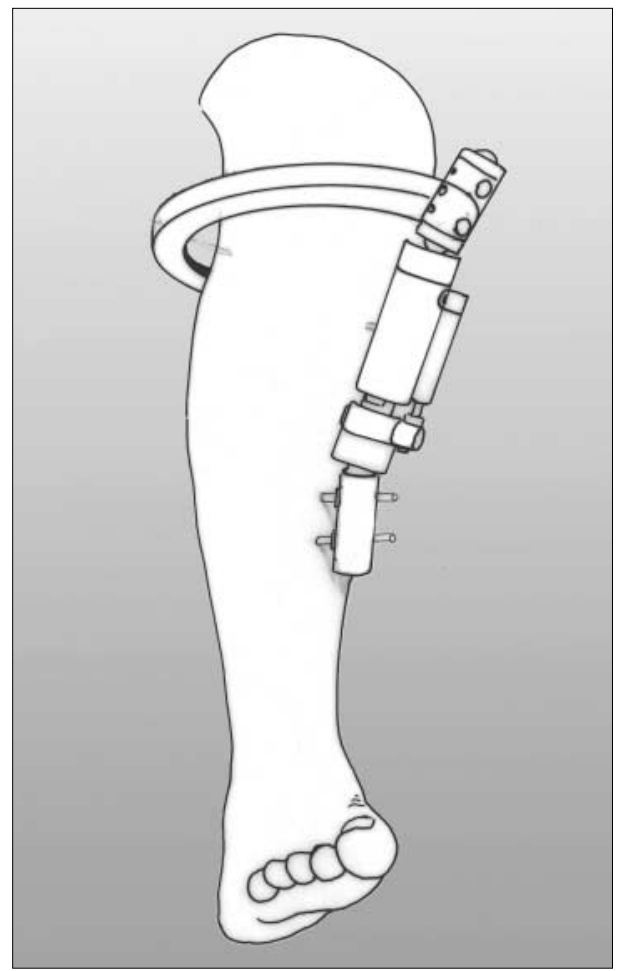

Fig. 3 The fracture was fixed with a hybrid external fixator

and the body of the external fixator was applied on the ring on the antero-medial aspect of the tibia. Two pin guides were insert- ed down to the skin, which was incised. The pin holes were predrilled with a $4.8-\mathrm{mm}$ drill bit, and two 5/6-mm tapered selftapping hydroxyapatite-coated pins were inserted $[19,20]$. The fixator was clamped to the pins. In 4 patients, 2 pins were also inserted in the lateral femoral diaphysis and a second fixator was applied across the knee to the tibial pins to permit immediate weight bearing.

Postoperative care consisted of immediate passive range of motion of the knee. Patients were discharged from the hospital between the 3rd and 15th day (mean 6 days) after surgery depending on their general condition. The patients with Gustilo grades II and III open fractures were checked weekly in the outpatient department. The other patients were seen monthly. Progressive weight bearing was allowed between the 8th and 12th week depending on the radiographic appearance of the callus. The external fixator was removed between 15 and 21 weeks after surgery (mean 17 weeks). All patients were evaluated by the clinical rating system of the Knee Society [21]. SPSS software package (Version 9.0, SPSS Inc., Chicago, IL, USA) was used to perform statistical analyses. All continuous data were expressed in terms of mean minimum and maximum value. The Wilcoxon non parametric test was used to calculate the difference of clinical rating system and range of motion over time.

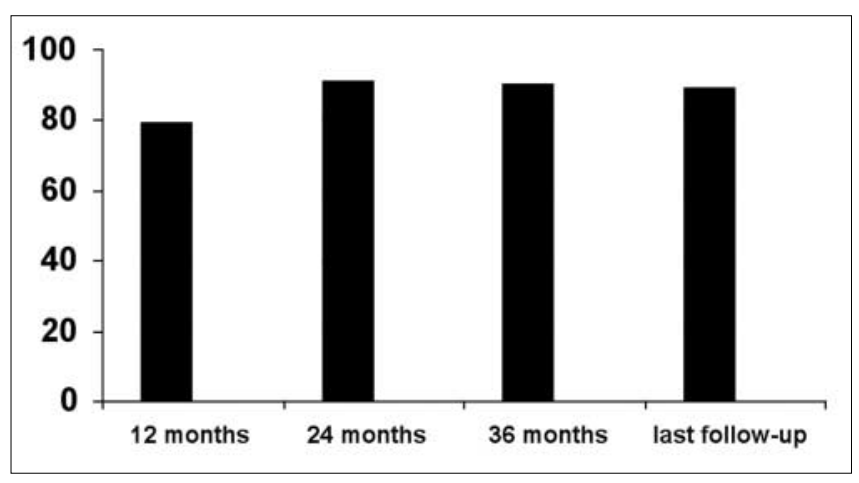

Fig. 4 Mean range of motion of the knee joints at each follow-up. No statistical differences were observed between the last available follow-up and 2 years follow-up. (p n.s.)

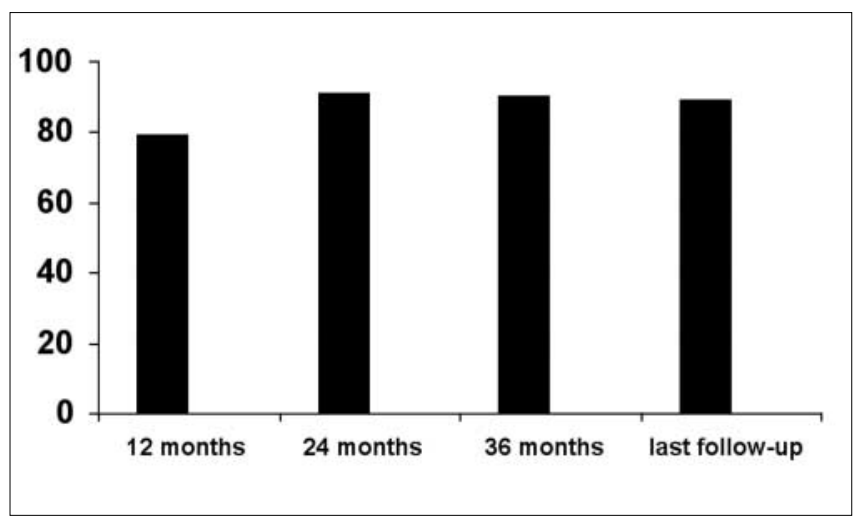

Fig. 5 Mean Knee society clinical score of the affected knee joints at each follow-up. No statistical differences were observed between the last available follow-up and 2 years follow-up. (p n.s.) 


\section{Results}

There were no intraoperative complications in the series. The external fixation system was tolerated for the entire treatment period in all cases. No pin or wire loosening or deep infections were observed. Two fractures resulted with angular deformity: one of $7^{\circ}$ in varus and the other one of $10^{\circ}$ in valgus, but in both cases the tibial plateau was adequately reducted and the axial deformity derived by the alignment of the metaphysis. In all cases the reduction of the epiphysis was adequate to regain the proper shape of tibial plateau. The average healing time was 24 weeks (range 18-29 weeks). In 1 patient a non-union occurred. This patient was later treated with open reduction and plate fixation. Patients with Gustilo grades II and III open fractures healed from both skeletal and soft tissue damage in an average time of 19.8 weeks (range 19-21 weeks). Two patients with knee deformity were treated with a second procedure consisting in tibial osteotomy 1 year after the trauma. Mean range of motion of the knee joint was $105^{\circ}\left(75-125^{\circ}\right)$ and mean Knee Society clinical score was 89 , twenty-five results were scored as excellent, 4 as good, 2 as fair and 1 as poor at last available followup of 48 month (range, 38-57 months) (Figs. 4, 5).

\section{Conclusions}

Closed reduction of tibial plateau fractures and fixation with 1 or 2 screws associated to a hybrid external fixator provides careful management of the soft tissue injury. The presence of extensive subcutaneous haemorrhage and bruising did not hinder percutaneous placement of the wires: use of wires avoids additional devitalisation of the bone as the periosteal and endosteal blood supply are not further compromised (Fig. 6). Marsh et al. [23] reported good results with a similar technique using monolateral external fixators, but observed loosening and infection of the proximal pins implanted in cancellous bone of the tibial epiphysis. In our experience, this can be avoided by using a hybrid frame. The use of a ring with tensioned wires near the joint permits epiphysial fixation of the tibia, reducing the risk of loosening and infection (Fig. 7). The utilisation of hydroxyapatite-coated screws according to Moroni et al. reduces the risk of both pin loosening and infection in the diaphysis [24, 25].

A potential disadvantage of this technique is the decreased accuracy of reduction of the articular surface compared with that obtained during open reduction. Koval et al. [15] demonstrated the difficulty in obtaining and assessing accurate reduction of compressed fragments with indirect techniques and fluoroscopic visualisation. We ob-
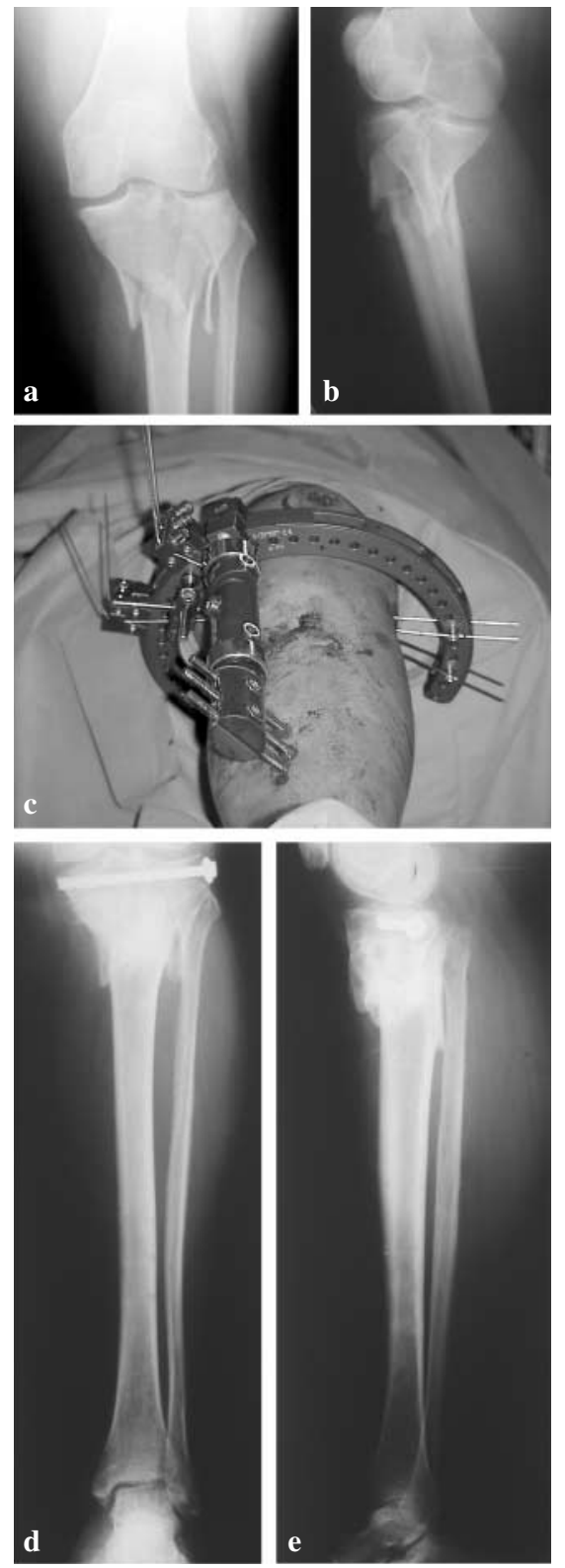

Fig. 6a, b Complex tibial plateau fracture in a 64-year-old patient. c Closed reduction with screws and hybrid external fixator. d, e Excellent results at 4-year follow-up

served inadequate reduction of the articular surface in 5 of our patients, and only 2 developed a secondary varus deformity that required surgical correction. This phenomenon was seen particularly in highly comminuted fractures. We believe that the disadvantage of a higher risk of insufficient reduction compared to open reduction and fixation is outweighed by the low risk of wound problems and septic arthritis. Furthermore, knee rehabilitation could be implemented immediately after surgery. We believe that these factors are responsible for optimal clinical long-term results. 

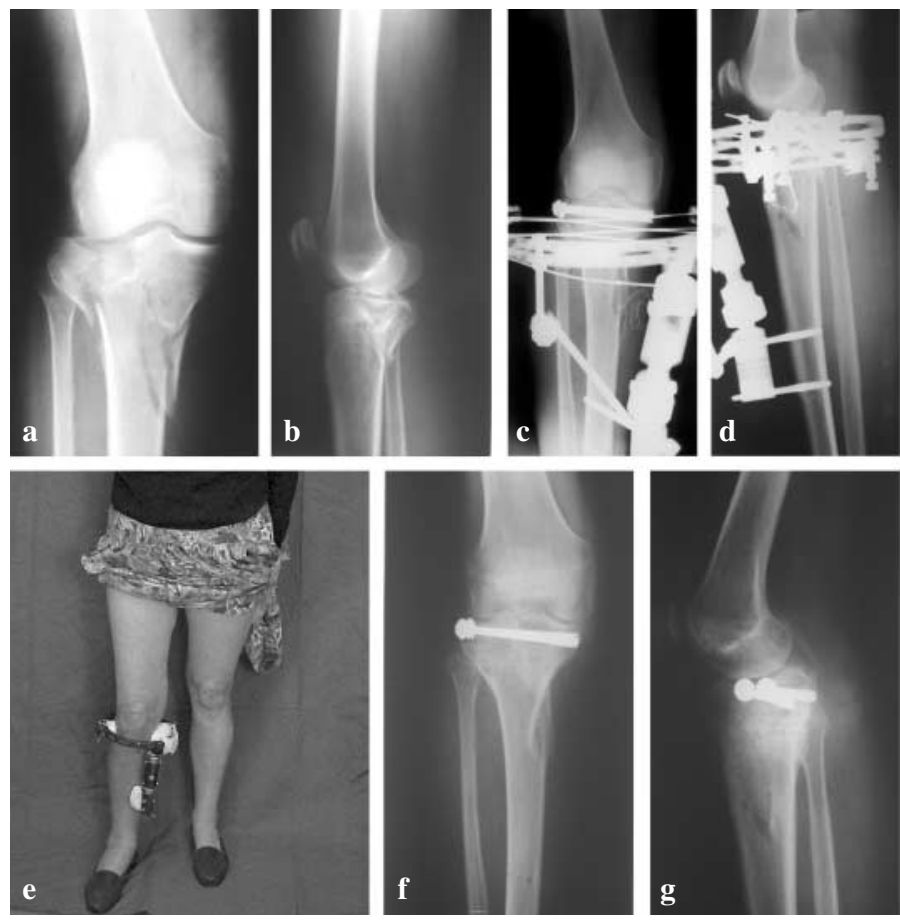

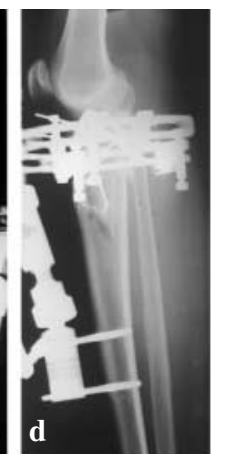

Fig. 7a, b Complex tibial plateau fracture in a 60-year-old patient. c, d Closed reduction with screws and hybrid external fixator. e Patient was allowed weight bearing 12 weeks after surgery. $\mathbf{f}, \mathbf{g}$ Excellent radiographic results at 4-year follow-up
Acknowledgements We wish to thank Alessandra Faldini and Monica di Ruscio, editorial assistants at the Department of
Endocrinology and Orthopaedic Trauma, University of Pisa, for their invaluable work in organising the data of the series.

\section{References}

1. Müller ME, Allgower M, Schneider R, Willenegger H (1981) Manuale dell'osteosintesi. Piccin (Ed), Padova, Italy

2. Schatzkler J, McBroom R, Bruce D (1979) The tibial plateau fractures. Clin Orthop 138:94-104

3. Brown GA, Sprague BL (1976) Cast brace treatment of plateau and bicondylar tibial plateau fractures. Clin Orthop 119:184-193

4. DeCoster TA, Nepola JV, El-Khoury GY (1988) Cast brace treatment of proximal tibia fractures: a ten-year follow up study. Clin Orthop 231:196-204

5. Delamarter R, Hohl M (1989) The cast brace and tibial plateau fractures. Clin Orthop 242:26-31

6. Drennan DB, Locher FG, Maylahn DJ (1979) Fractures of the tibial plateau: treatment by closed reduction and spica cast. J Bone Joint Surg 61A:989-995

7. Sarmiento A, Kinman PB, Latta LL, Eng P (1979) Fractures of the proximal tibia and tibial condyles: a clinical and laboratory comparative study. Clin Orthop 145:136-145
8. Scotland T, Wardlaw D (1981) The use of cast bracing as treatment for fractures of the tibial plateau. J Bone Joint Surg 63B:575-578

9. Stannard JP, Wilson TC, Volgas DA, Alonso JE (2004) The less invasive stabilization system in the treatment of complex fractures of the tibial plateau: short-term results. J Orthop Trauma 18:552-558

10. Egol KA, Su E, Tejwani NC, Sims SH, Kummer FJ, Koval KJ (2004) Treatment of complex tibial plateau fractures using the less invasive stabilization system plate: clinical experience and a laboratory comparison with double plating. J Trauma 57:340-346

11. Sirkin MS, Bono CM, Reilly MC, Behrens FF (2000) Percutaneous methods of tibial plateau fixation. Clin Orthop 375:60-68

12. Young MJ, Barrack RL (1994) Complications of internal fixation of tibial plateau fractures. Orthop Rev 23:149-154
13. Barei DP, Nork SE, Mills WJ, Bradford Henley M, Benirschke SK (2004) Complications associated with internal fixation of high-energy bicondylar tibial plateau fractures utilizing a two-incision technique. J Orthop Trauma 18:649-657

14. Duwelius PJ, Connoly JF (1988) Closed reduction of tibial plateau fractures: a comparison of functional and roentgenographic end results. Clin Orthop 230:116-126

15. Koval KJ, Sanders R, Borrelli $\mathrm{J}$ et al (1992) Indirect reduction and percutaneous screws fixation of displaced tibial plateau fractures. J Orthop Trauma 6:340-346

16. Lobenhoffer P, Schulze M, Gerich T, Lattermann C, Tscherne H (1999) Closed reduction/percutaneous fixation of tibial plateau fractures: arthroscopic versus fluoroscopic control of reduction. J Orthop Trauma 6:426-431

17. Watson JT (2001) Hybrid external fixation for tibial plateau fractures. Am J Knee Surg 14:135-140 
18. Gaudinez RF, Mallik AR, Szporn M (1996) Hybrid external fixation of comminuted tibial plateau fractures. Clin Orthop 328:203-210

19. Watson JT, Coufal C (1998) Treatment of complex lateral plateau fractures using Ilizarov techniques. Clin Orthop 353:97-106

20. Kumar A, Whittle AP (2000) Treatment of complex (Schatzkler type VI) fractures of the tibial plateau with circular wire external fixation: retrospective case review. J Orthop Trauma 14:339-344
21. Insall JN, Dorr LD, Scott RD, Scott WN (1989) Rationale of the Knee Society clinical rating system. Clin Orthop Relat Res 248:13-14

22. Gustilo RB (1987) Current concepts in management of open fractures. Instr Course Lect 36:357-369

23. Marsh JL, Smith ST, Do TT (1995) External fixation and limited internal fixation for complex fractures of the tibial plateau. J Bone Joint Surg 77A:661-673
24. Magyar G, Toksvig-Larsen S, Moroni A (1997) Hydroxyapatite coating of threaded pins enhances fixation. J Bone Joint Surg 79B:487-489

25. Moroni A, Aspenberg P, Toksvig Larsen S, Falzano G, Giannini S (1998) Enhanced fixation with hydroxyapatite pins. Clin Orthop 364:171-177 